\title{
Search for supersymmetry in lepton/photon(s)/ $b$-jets and missing transverse momentum final states with the ATLAS detector
}

Helen HAYWARD*i

University of Liverpool

E-mail: Helen.haywardecern.ch

\begin{abstract}
Channels with one or several leptons or photons and high missing transverse momentum (and potentially high $p_{T}$ jets or $b$-jets) offer potentially fruitful signatures with which to search for supersymmetry at the LHC. In this paper, the most recent results on these channels are given for data collected by the ATLAS experiment in 2011.
\end{abstract}

The 2011 Europhysics Conference on High Energy Physics-HEP 2011,

July 21-27, 2011

Grenoble, Rhône-Alpes France

*Speaker.

$\dagger$ On behalf of the ATLAS collaboration 


\section{Introduction}

Supersymmetry (SUSY) [1] is a candidate for a theory to describe physics beyond the Standard Model (SM). It introduces a symmetry between fermions and bosons, resulting in a SUSY partner (sparticle) with identical quantum numbers (differing by half a unit of spin) for each SM particle. As none of these sparticles have been observed, SUSY must be a broken symmetry if realised in nature. Assuming R-parity conservation[3], sparticles have to be produced in pairs. These would then decay through cascades involving other sparticles until the lightest SUSY particle (LSP) is produced. The LSP is stable and escapes detection, manifesting itself in missing transverse energy $\left(E_{T}^{m i s s}\right)$ in the event. The experimental signature of SUSY events is largely determined by the character of the next-to-lightest SUSY particle (NLSP).

In this paper we discuss the search for SUSY using leptons, photons and $b$-jets in the final state. The most recent results on these channels from the ATLAS experiment[2] are presented.

\section{The ATLAS Detector}

The ATLAS detector is designed as a general purpose detector with a a symmetric cylindrical geometry, providing nearly $4 \pi$ solid angle coverage. Closest to the beam pipe are tracking detectors using silicon $(|\eta|<2.5)$ and straw-tube detector technologies $(|\eta|<2.0)^{1}$, located inside a superconducting solenoid providing a $2 \mathrm{~T}$ magnetic field. Further out from the interaction point lies the calorimeter system, consisting of liquid-argon (LAr) sampling calorimeter and iron-scintillator tile calorimeter to provide electromagnetic and hadronic coverage. The outermost detector is a muon spectrometer consisting of three large super-conducting toroids, a system of precision tracking chambers and detectors for triggering.

\section{Search for SUSY with exactly one high $p_{T}$ lepton.}

If charginos $\left(\tilde{\chi}^{ \pm}\right)$are produced in the SUSY chain, they can produce a high-momentum lepton. An analysis[4] was designed to search for such events with only one lepton, such that it can be combined with other channels ( $0,2,3$ lepton channels) and is not optimised for any particular model of SUSY.

A dataset based on an integrated luminosity of $165 \mathrm{pb}^{-1}$ from proton-proton collisions collected in 2011 in by the ATLAS experiment at a center-of-mass energy of $7 \mathrm{TeV}$ was used. Events were selected, after the application of basic data quality requirements, by single leptons triggers $(e$ or $\mu$ ). Events containing exactly one well reconstructed and isolated electron or muon with transverse momentum $p_{T}>20 \mathrm{GeV}$ were selected. The events were also required to have 3 good jets, with $|\eta|<2.8$ of $p_{T}>60 \mathrm{GeV}$ for the leading jet, and $p_{T}>25 \mathrm{GeV}$ for the second and third jets. Large mismeasurement of the jet transverse momenta is avoided by requiring the $E_{T}^{\text {miss }}$ vector to not be aligned with any of the three leading jets $\left(\Delta \phi\left(\overrightarrow{j e t}_{i}, \vec{E}_{T}^{m i s s}\right)>0.2\right)$. In addition the transverse

\footnotetext{
${ }^{1}$ ATLAS uses a right-handed coordinate system with its origin at the nominal interaction point (IP) in the centre of the detector and the $z$-axis along the beam pipe. The $x$-axis points from the IP to the centre of the LHC ring, and the $y$ axis points upward. Cylindrical coordinates $(r, \phi)$ are used in the transverse plane, $\phi$ being the azimuthal angle around the beam pipe. The pseudorapidity is defined in terms of the polar angle $\theta$ as $\eta=-\ln \tan (\theta / 2)$.
} 
mass $\left(m_{T}\right)$ and $E_{T}^{\text {miss }}$ were required to be larger than 100 and $125 \mathrm{GeV}$ respectively. The two final cuts that define the signal region are $E_{T}^{\text {miss }} / m_{\text {eff }}>0.25$ and $m_{\text {eff }}>500 \mathrm{GeV}$, where $m_{\text {eff }}$ is the scalar sum of $E_{T}^{\text {miss }}$ and the transverse momenta of the three leading jets.

The main SM backgrounds are $t \bar{t}$ and $W+$ jet events which are estimated with data-driven techniques. The number of observed events in all three analyses has been found in agreement with the SM predictions, as shown in table 1.

Table 1: Summary of the observed and expected event yields in events with a single electron or muon.

\begin{tabular}{lrr}
\hline & Electron Channel & Muon Channel \\
\hline Observed Events & 10 & 12 \\
\hline Estimated Top events & $8.7 \pm 3.6$ & $7.2 \pm 2.7$ \\
Estimated W/Z events & $4.6 \pm 2.8$ & $5.0 \pm 2.6$ \\
Estimated QCD events & $1.12_{-0.4}^{+0.7}$ & $0.0_{-0.0}^{+0.5}$ \\
\hline Total Background & $14.5 \pm 5.2$ & $12.2 \pm 3.8$ \\
\hline
\end{tabular}

From the number of observed events and predicted background, 95\% CL upper limits on the observed number of signal events in the electron and muon channels of 6.8 and 8.8 were made respectively. The results are interpreted as limits for a grid of signal models in the $m_{0}-m_{1 / 2}$ plane, as shown in figure 1 .

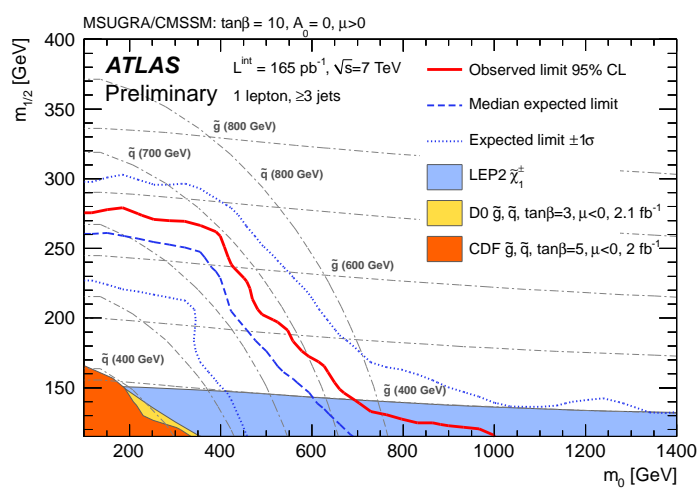

Figure 1: Observed and expected 95\% CL exclusion limits, as well as the +- 1 sigma variation on the expected limit, in the combined electron and muon channels. Results are obtained with the PCL technique. The plots also show the published limits from CDF, D0, and the results from the LEP experiments.

\section{Search for Diphoton events with large $E_{T}^{\text {miss }}$}

In Gauge Mediated Supersymmetry breaking (GMSB)[5] the LSP is the Gravitino $(\tilde{G})$ and the NLSP is commonly the lightest neutralino $\tilde{\chi}^{0}$, which if the SUSY partner of the U(1) gauge boson (the "bino"), the final decay in the cascade is $\tilde{\chi}^{0} \rightarrow \gamma \tilde{G}$. The two susy cascades result in a final state $\gamma \gamma+E_{T}^{\text {miss }}+X$, where $X$ represents SM particles emitted in the prompt cascade decays. 
From the full data sample collected in 2010 of $36 \mathrm{pb}^{-1}$, photon triggers were used to select events. 762 events had two good photons $\left(E_{T}>30,20 \mathrm{GeV}\right)$, although none of these events were found in the signal region of $E_{T}^{\text {miss }}>125 \mathrm{GeV}$ [6]. Data-driven techniques were used to estimate an expected background of $0.10 \pm 0.04$ (stat) \pm 0.05 (sys) events. The number of events in the signal region from any physics beyond that described by the SM, was found to be less than 3.0 at 95\% CL. This number corresponds to 95\% CL limits on the production cross-sections of $\sigma<0.38-0.65 \mathrm{pb}$ in the GGM model $\left(m_{\tilde{\chi}_{1}^{0}}=150 \mathrm{GeV}, m_{\tilde{g}}=400-800 \mathrm{GeV}\right)$. This estimate was used, together with the observation of zero events, to place a 95\% CL upper limit on the number of events from new physics in the signal region as shown in figure 2.

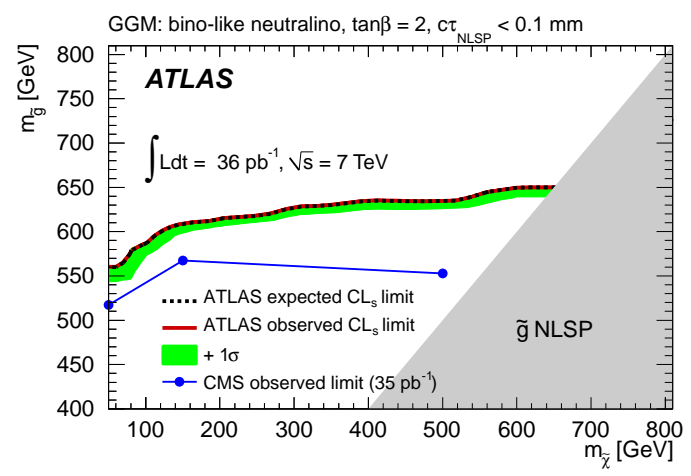

Figure 2: Expected and observed $95 \%$ CL lower limits on the gluino mass as a function of the neutralino mass in the GGM model with a bino-like lightest neutralino as NLSP (the grey area indicates the region where the NLSP is the gluino, which was not considered here). The other sparticle masses are fixed to 1.5 TeV. Further model parameters are tan beta $=2$ and $\mathrm{c}$ tauNLSP $<0.1 \mathrm{~mm}$. The observed limit and the -1 sigma expected error band are degenerate with the expected limit.

\section{Search for SUSY with $b$-jets and no leptons}

SUSY is searched for in final states involving $E_{T}^{\text {miss }}$, energetic jets, of which one must be identified as a $b$-jet and no isolated leptons ( $e$ or $\mu$ ). A total of $0.83 \mathrm{fb}^{-1}$ of data is considered[7]. Events are triggered with a high $p_{T}$ jet and large $E_{T}^{\text {miss }}$. They are required to have at least one jet with $p_{T}>130 \mathrm{GeV}$, two softer jets with $p_{T}>50 \mathrm{GeV}, E_{T}^{\text {miss }}>130 \mathrm{GeV}$ and $E_{T}^{\text {miss }} / m_{\text {eff }}>0.25$. Four signal regions are defined in order increase signal sensitivity for the models considered. They are characterised by the number of identified $b$-jets and $m_{e f f}$ and presented in table 2 with the observed and expected number of events they contained. These numbers were used to produce 95\% CL upper limits on the number of events and effective cross-section from new physics. These results are interpreted in terms of two phenomenological MSSM scenarios, as shown in figure 3. In the first of these, the $\tilde{b}_{1}$ is the lightest squark $\left(m_{\tilde{g}}>m_{\tilde{b}_{1}}>m_{\tilde{\chi}^{0}}\right)$ and is produced via gluino mediated processes or via direct pair production and decay exclusively to $\tilde{b}_{1} \rightarrow b \tilde{\chi}_{1}^{0}$. The second scenario is defined in the context of the general simplified models[8] where the gluinos decay into $b \tilde{b} \tilde{\chi}_{1}^{0}$ final states. 
Table 2: Summary of the observed and expected event yields in the four signal regions. Systematic uncertainties for the SM predictions are given. Also shown are the 95\%C.L. upper limits on contributions from new physics using the $C L_{s}$ method and on the effective cross-section for new physics.

\begin{tabular}{|c|c|c|c|c|}
\hline \multirow{2}{*}{ Signal Region } & $1 b$-jet & $1 b$-jet & $2 b$-jet & $2 b$-jet \\
\hline & $m_{e f f}>500 \mathrm{GeV}$ & $m_{e f f}>700 \mathrm{GeV}$ & $m_{e f f}>500 \mathrm{GeV}$ & $m_{e f f}>700 \mathrm{GeV}$ \\
\hline Observed events & 361 & 63 & 76 & 12 \\
\hline Est. Top events & $221_{-68}^{+82}$ & $37_{-12}^{+15}$ & $55_{-22}^{+25}$ & $7.8_{-2.9}^{+3.5}$ \\
\hline Est. W/Z events & $121 \pm 61$ & $31 \pm 19$ & $20 \pm 12$ & $5 \pm 4$ \\
\hline Est. QCD events & $15 \pm 7$ & $1.9 \pm 0.9$ & $3.6 \pm 1.8$ & $0.5 \pm 0.3$ \\
\hline Total Background & $356_{-92}^{+103}$ & $70_{-22}^{+24}$ & $\begin{array}{l}79_{-25}^{+28} \\
\end{array}$ & $13.0_{-5.2}^{+5.6}$ \\
\hline $95 \%$ C.L. $N_{\text {events }} C L_{s}$ & 140 & 51 & 65 & 14 \\
\hline $95 \%$ C.L. $\sigma_{e f f}(\mathrm{pb}) C L_{s}$ & 0.288 & 0.061 & 0.078 & 0.017 \\
\hline
\end{tabular}
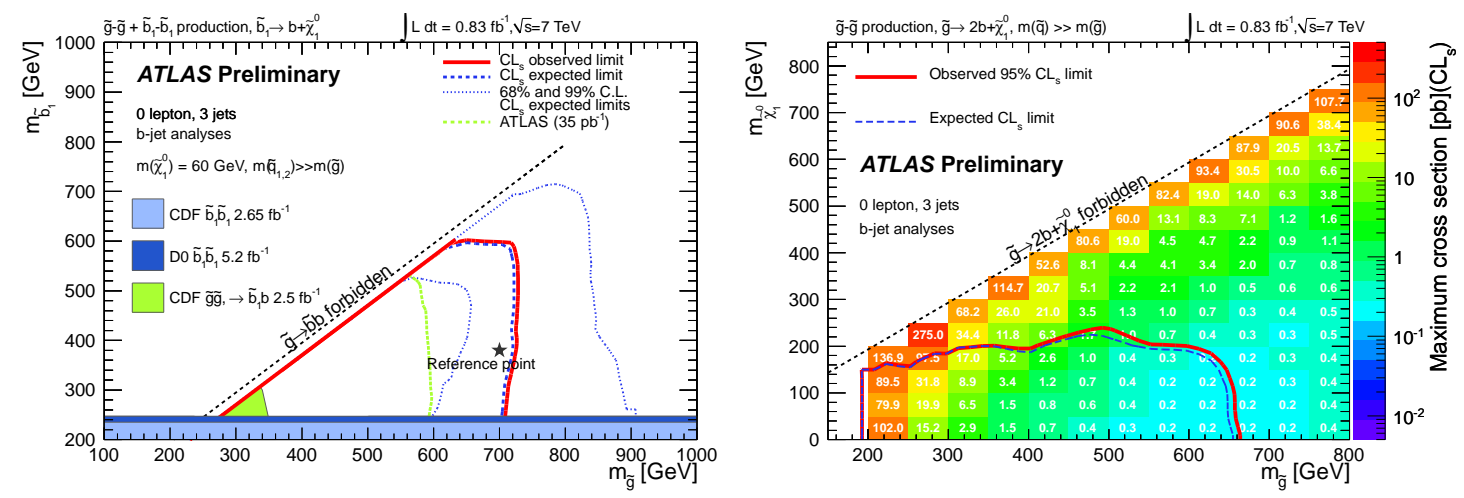

Figure 3: The plot on the left shows the observed and expected $95 \%$ C.L. exclusion limits in the gluinosbottom mass plane. Also shown are the $68 \%$ and $99 \%$ C.L. expected exclusion curves. For each point in the plot, the signal region selection providing the best expected limit is chosen. The neutralino mass is set to $60 \mathrm{GeV}$. The result is compared to previous results from ATLAS and CDF searches which assume the same gluino-sbottom decays hypotheses. Exclusion limits from the CDF and D0 experiments on direct sbottom pair production are also shown. The plot on the right shows the 95\% C.L. upper cross section limits in pb and observed and expected limit contours in the gluino-neutralino (LSP) mass plane for gluino masses above $200 \mathrm{GeV}$. For each scenario, the signal region selection providing the best expected limit is chosen.

\section{Summary}

Searches for SUSY with photons, leptons and $b$-jets have been performed at ATLAS experiment using data collected in 2010 and the beginning of 2011. The observed number of events in the signal regions shows agreement with the expected background from SM processes. Limits are placed on the number of events from new physics. 


\section{References}

[1] Y. A. Golfand and E.P. Likhtman, JETP lett. 13 (1971) 323.

[2] ATLAS Collaboration, JINST 3 (2008) S08003.

[3] P. Fayet, Phys. Lett. B 69 (1977) 489; G. R. Farrar and P. Fayet, Phys. Lett. B 76 (1978) 575.

[4] ATLAS Collaboration, ATLAS-CONF-2011-090 (2011).

[5] L. Alvarez-Gaume, M. Claudson, and M. Wise, Nucl.Phys. B207 (1982) 96.

[6] ATLAS Collaboration, Eur. Phys. J. C. 71 (2011) 10.

[7] ATLAS Collaboration, ATLAS-CONF-2011-098 (2011).

[8] D. E. A. Alves, arXiv:1105.2838 [hep-ph] (2011). 